\title{
Dinámica espacio-temporal de 13 especies de peces nativos en un ecotono lacustre-fluvial de la Cuenca del Río Valdivia (Chile)
}

\section{Spatio-temporal dynamics of 13 species of native fish in a lake - river ecotone in the Valdivia River Basin (Chile)}

\author{
Claudio Valdovinos ${ }^{1 *}$, Evelyn Habit ${ }^{2}$, Alfonso Jara ${ }^{2}$, Priscila Piedra ${ }^{2}$, Jorge González ${ }^{2}$ \& Jacqueline \\ SALVO ${ }^{1}$
}

${ }^{1}$ Laboratorio de Biodiversidad y Conservación de Recursos Acuáticos, Centro de Ciencias Ambientales EULA-Chile, Universidad de Concepción. Casilla 160-C, Concepción, Chile.

${ }^{2}$ Laboratorio de Ecología y Conservación de Peces, Unidad de Sistemas Acuáticos, Centro de Ciencias Ambientales EULAChile, Universidad de Concepción. Casilla 160-C, Concepción, Chile.

*Email: cvaldovi@udec.cl

\begin{abstract}
RESUMEN
Los ecotonos transicionales entre lagos y ríos han recibido menor atención que aquellos de ecosistemas terrestres, y pueden reunir características particulares de gran importancia para la conservación de la biodiversidad dulceacuícola. Con el objeto de evaluar lo anterior, se estudió la dinámica espacio-temporal de las abundancias y tallas, de poblaciones de 13 especies de peces nativos chilenos, presentes en la zona ecotonal Lago Riñihue - Río San Pedro, ubicada en la cuenca hidrográfica del río Valdivia (Chile). Se muestrearon mensualmente 35 sitios localizados en tres zonas hidromorfológicas del área de estudio (número de sitios por zona: $\mathrm{M} 1=9, \mathrm{M} 2=17, \mathrm{M} 3=9$ ). Los muestreos se llevaron a cabo el año 2006. De manera complementaria, se realizaron muestreos en todos los sitios los años 2007 (enero - febrero, junio - agosto y diciembre) y 2008 (enero - marzo). Los resultados de este estudio muestran la presencia de un ecotono muy dinámico tanto espacial como temporalmente, apoyando la hipótesis que estas zonas transicionales poseen características especiales que deben ser tomadas en consideración para la conservación de la biodiversidad.
\end{abstract}

Palabras claves: Biodiversidad, Ecotonos, Ecología, Ictiofauna, Río San Pedro, Chile.

\begin{abstract}
The transitional ecotones between lakes and rivers have received less attention than those of terrestrial ecosystems and can meet particular characteristics of great importance for freshwater biodiversity conservation. In order to evaluate this, we studied the spatio-temporal dynamics of the abundances and body sizes of the populations of 13 species of Chilean native fishes, in the ecotonal area Riñihue Lake - San Pedro river, located in the Valdivia river basin (Chile). Thirty five sites were sampled monthly, located in three hydromorphological zones of the study area (number of sites by area: M1 = 9, M2 = 17 M3 =9). The results of this study show the presence of a dynamic ecotone both spatially and temporally, supporting the hypothesis that these transitional zones have special characteristics that must be taken into account for conservation of biodiversity.
\end{abstract}

KeYwords: Biodiversity, Ecotones, Ecology, Ichthyofauna, San Pedro river, Chile.

\section{INTRODUCCIÓN}

A pesar de los esfuerzos de investigación sobre la biología y ecología de peces dulceacuícolas de Chile, realizados especialmente en las últimas dos décadas, el conocimiento que se tiene de la variabilidad espacio-temporal de las poblaciones es todavía limitado. La mayor parte de los estudios se ha centrado en las fluctuaciones estacionales a lo largo de un año (Habit et al. 2006a). Son escasos los estudios de este tipo que se han extendido por más de un ciclo anual (ver Habit et al. 2007). Por otra parte, hasta el momento no han existido en Chile estudios, que con una elevada resolución temporal, e.g. mensual, analicen el comportamiento de todas las poblaciones de peces nativos existentes en un tramo de río, considerando además su variabilidad espacial. Este tipo de estudios son relevantes ya 
que permiten reconocer cuáles son las variaciones naturales tanto espaciales como temporales de largo plazo, las cuales constituyen temas centrales en el estudio de los ecosistemas fluviales (Allan \& Castillo 2007).

Los ecotonos de agua dulce se caracterizan por ser ecosistemas únicos, albergar altos niveles de diversidad biológica y ser sensibles a las alteraciones antropogénicas. Además cumplen diversas funciones como corredores biológicos y membranas semipermeables que controlan el flujo de materia y energía entre unidades ecológicas adyacentes (Naiman \& Décamps 1997). El grado de particularidad de ellos, está dado por la escala-dependencia y por la fuerza de interacción entre ambas unidades, es decir, mientras mayor es el grado de contraste, mayor es la fuerza de interacción entre ellas. Son estas particularidades, las que convierten a los ecotonos en ecosistemas sensibles a los cambios ambientales (Dudgeon et al. 2006). El estudio de los ecotonos se ha centrado en ecosistemas terrestres, con un fuerte enfoque en las transiciones vegetacionales. A diferencia de los ecotonos dulceacuícolas, los cuales han recibido menos atención, a pesar de que su ocurrencia es evidente a través de diversas escalas temporales (Heegaard et al. 2006) y espaciales (Statzner \& Higler 1986). Uno de los ecotonos más estudiados en los ecosistemas dulceacuícolas, es la transición acuática terrestre o zona ribereña. Este ecotono, es el resultado de la transición entre los límites de parches discretos de hábitat que surgen de la heterogeneidad de los ecosistemas fluviales (Naiman et al. 1988). También han recibido atención los ecotonos existentes entre el agua superficial y el hiporreos, al igual que los estuarios. Las transiciones lago-río han recibido menos atención, y pueden reunir características particulares de gran importancia para la gestión y conservación de los ecosistemas lacustres y fluviales (Samways \& Stewart 1997; Willis \& Magnuson 2000; Jones 2010; Rader et al. 2011). La cuenca hidrográfica del río del Valdivia localizada en el centro-sur de Chile, corresponde a una de las más diversas en ictiofauna nativa del país, y dado su escaso grado de intervención humana, la hacen un sitio adecuado para el estudio de las variaciones espacio - temporales de estos organismos, especialmente en áreas ecotonales de la transición entre sistemas lacustres y fluviales. El río Valdivia tiene sus principales afluentes en el área cordillerana, correspondientes a los ríos Quinchilca y San Pedro. Este último río se origina al término de una serie de ocho lagos que comienzan al lado oriental de la Cordillera de los Andes, y reúne una ictiofauna con un amplio espectro de estrategias de vida. Muchas de las especies presentes en este río poseen complejos patrones poblacionales (Victoriano et al. 2011), incluso dentro de una misma especie (e.g. Galaxias maculatus), constituyendo distintas unidades naturales que se desarrollan diferencialmente entre las distintas zonas del río (ver García et al. 2011; Colin et al. 2011; Montoya et al. 2011). En el presente estudio se describe y analiza las dinámica espacio-temporal de las poblaciones de 13 especies de peces nativos, en una zona ecotonal correspondiente al desagüe del Lago Riñihue y el Río San Pedro (ver Campos et al. 1978). Este estudio representa la primera aproximación en Chile para conocer las características y dinámica de los ecotonos lacustresfluviales, y proporciona antecedentes que serán de utilidad para un adecuado manejo conservativo de la biodiversidad de tales zonas.

\section{MATERIALES Y MÉTODOS}

Área de ESTUdio

El área de estudio correspondió al ecotono constituido por la desembocadura del lago Riñihue y el Río San Pedro (3946' - 39 $51^{\circ}$ 'S), dentro de la cuenca hidrográfica del río Valdivia $\left(10.244 \mathrm{~km}^{2}\right)$. Esta cuenca la compone una cadena de ocho lagos de origen glaciar y características oligotróficas que nace en Argentina, y está compuesta por los siguientes lagos: Lácar, Pirehueico, Neltume, Pellaifa, Calafquén, Pullinque, Panguipulli y Riñihue (Thomasson 1963; Campos et al. 1978). Este último lago corresponde al que da origen al río San Pedro (Fig.1). Dado que hasta el momento la cuenca no presenta intervención humana significativa, presenta un comportamiento hidrológico regulado por la cadena de lagos localizados aguas arriba, con un régimen natural de caudales altamente dependiente de la pluviosidad (Eula 2007).

Se estudió el río San Pedro en todo su recorrido de $40 \mathrm{~km}$, desde el desagüe del Lago Riñihue (39 $46^{\prime}$ 'S; $72^{\circ} 27^{\prime} \mathrm{W}$ ), hasta $0,5 \mathrm{~km}$ aguas arriba de su confluencia con el río Quinchilca ( $\left.39^{\circ} 51^{\prime} \mathrm{S} ; 7^{\circ} 27^{\prime} \mathrm{W}\right)$. En él se identifican tres macrozonas hidrogeomorfológicas que fueron estudiadas (Tabla 1; Fig. 1). La primera (M1) corresponde al desagüe del lago Riñihue e inicio del río San Pedro (hasta sector Piedra Blanca), que es la zona de mayor profundidad (hasta $25 \mathrm{~m}$ ), con baja velocidad de la corriente, y con fondos de sustratos arcillosos consolidados. Este tramo incluye principalmente sectores lacustres de menor profundidad, incorporando también $1,5 \mathrm{~km}$ de río, que corresponden a los inicios del San Pedro. La segunda macrozona (M2), es un área con mayor pendiente y de laderas abruptas, donde el cauce presenta mayor velocidad de corriente, generando hábitats de rápidos y con riberas rocosas. Este tramo es el de mayor extensión, es típicamente no-aluvial con una longitud aproximada de $30 \mathrm{~km}$, y cubre aproximadamente el $75 \%$ del cauce del río San Pedro. Debido a la pendiente existen mayores velocidades de escurrimiento que en M1, observándose secuencias de rápidos profundos y pozas, en los cuales prevalecen los sustratos de bolones, con muchos bloques de grandes dimensiones. En algunos tramos de M2, hay una angosta planicie de inundación, poco desarrollada (este tramo se localiza entre el sector de Los Bajos hasta 
Chacaipulli). Por último, la zona donde el río ingresa en el valle (M3), se caracteriza por una menor pendiente que en $\mathrm{M} 2$ y la presencia hábitats mixtos entre rápidos y pozas (principalmente someras). Esta zona corresponde a un tramo de río aluvial, con secuencias de pozones y rápidos. En esta zona, el río no escurre encajonado como en M2, sino que muestra una amplia planicie de inundación, de hasta $600 \mathrm{~m}$ de ancho en su ingreso al Valle Central. Este tramo de $8,5 \mathrm{~km}$ de longitud se ubica desde el sector del Balsadero hasta aproximadamente $200 \mathrm{~m}$ aguas abajo de la Confluencia con el río Quinchilca. En los ca. $40 \mathrm{Km}$ de este río, y abarcando las tres áreas hidrogeomorfológicas (M1M3), se establecieron 35 sitios de muestreo, ubicados desde el lago Riñihue hasta la confluencia con el río Quinchilca (Fig. 1, Tabla 1).

\section{MueSTREO Y ANÁLISIS DE DATOS}

Se muestrearon con una frecuencia mensual un total de 35 sitios (Fig. 1, Tabla 1), distribuidas en las tres macrozonas estudiadas (M1-M3). Debido a la extensión y complejidad del área de estudio, fueron seleccionados en base a la representatividad de hábitats, nueve sitios de muestreo en M1, diecisiete en M2 y nueve en M3. Todos los sitios fueron muestreados mensualmente a lo largo del año 2006. De manera complementaria, se realizaron muestreos en todos los sitios los años 2007 (enero - febrero, junio - agosto y diciembre) y 2008 (enero - marzo). Desde un punto de vista hidrológico, es importante destacar que el año 2006 fue un año lluvioso, en contraste con los años 2007 y 2008 que fueron años secos.

El diseño de muestreo de peces consideró un tramo de estudio de 25 a $200 \mathrm{~m}$ de longitud, estratificado por microhábitats (Maddock 1999). El esfuerzo de muestreo se concentró en ambientes ribereños someros y profundos (máximo $1 \mathrm{~m}$ ),

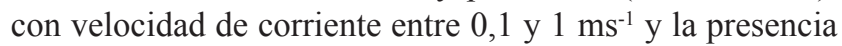
de sustratos variables, en donde se encuentran la mayoría de los peces de los sistemas fluviales (Arunachalam 2000). La captura de peces se realizó en las zonas ribereñas y pozones del río mediante pesca eléctrica (LR-24 Smith \& Root). Cada individuo capturado fue anestesiado mediante solución anestésica diluida en agua (BZ-20®, $1 \mathrm{~mL}: 5 \mathrm{~L}$ ), y medido con ictiómetro de $0,1 \mathrm{~cm}$ de precisión, determinando la longitud estándar $(L e)$. Posteriormente, y una vez recuperada su movilidad éstos fueron liberados a su ambiente.

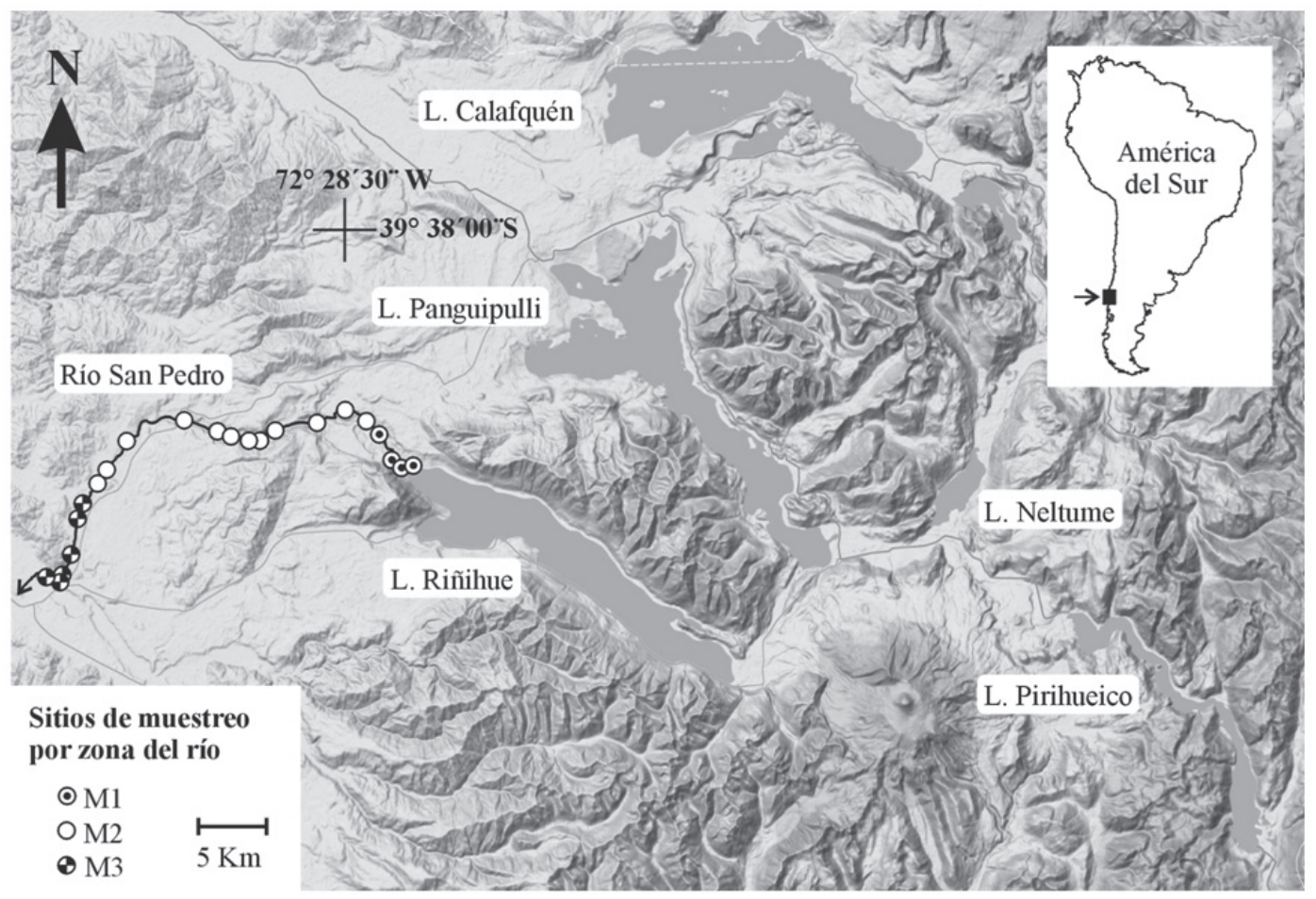

Figura 1. Área de estudio y localización de los sitios de muestreo en las tres macrozonas del Río San Pedro (Chile).

FIGURE 1. Study area and location of sampling sites conducted in the three macrozones of the San Pedro River (Chile). 
Para estudiar la dinámica de las abundancias de las poblaciones de las especies nativas, se confeccionaron matrices de abundancia basadas en Captura por Unidad de Esfuerzo (CPUE) para hacerlos comparables. Se estimó CPUE como $N 100 / t A$, donde $\mathrm{N}$ corresponde al número de individuos, $t$ al tiempo de pesca en horas y $A$ al área muestreada en $\mathrm{m}^{2}$. Para conocer la dinámica espacial de la abundancia de cada especie, se analizó la variación en la abundancia a través de las tres macrozonas identificadas a través del área de estudio (M1-M3). Con el objeto de estudiar la dinámica temporal de la abundancia de cada especie, se analizó la variación mensual en un ciclo anual. De manera complementaria y con la finalidad de identificar posibles patrones de reclutamiento, se estudiaron las tendencias de las tallas $(L e)$ de cada especie a lo largo del período de estudio.

TABla I. Puntos de muestreo de cada macrozona en el río San Pedro y sus respectivas coordenadas (UTM) y pendiente (\%).

TABLE I. Sample sites trough San Pedro River, indicating the macrozone which belong and their geographical coordinates (UTM) and slope $(\%)$.

\begin{tabular}{|c|c|c|c|c|c|}
\hline MACROZONA & Nombre del Sitio & Código & $\begin{array}{c}\text { UTM } \\
\text { X }\end{array}$ & $\begin{array}{c}\text { UTM } \\
\mathrm{Y} \\
\end{array}$ & $\begin{array}{c}\text { Pendiente } \\
(\%)\end{array}$ \\
\hline 1 & Isla Grande & IG & 718328 & 5594046 & 0,28 \\
\hline “ & Hotel Riñimapu & $\mathrm{HO}$ & 717859 & 5594106 & 0,50 \\
\hline “ & Desembocadura Catalina & DC & 717642 & 5594034 & 0,39 \\
\hline “ & Piedra Blanca & $\mathrm{PB}$ & 717526 & 5594451 & 0,50 \\
\hline 2 & Los Bajos & LB & 716917 & 5595892 & 1,00 \\
\hline “ & Carmen del Trafún Este & $\mathrm{TE}$ & 715426 & 5596941 & 1,30 \\
\hline 6 & Carmen del Trafún Oeste & TO & 712171 & 5597029 & 1,50 \\
\hline “ & Champulli & $\mathrm{CH}$ & 708350 & 5596457 & 1,00 \\
\hline “ & Forestal Maitén & FM & 707795 & 5596191 & 1,20 \\
\hline “ & El Maitén & MA & 707240 & 5596014 & 1,30 \\
\hline “ & La Quinta & LQ & 706945 & 5595750 & 1,30 \\
\hline “ & Nuevo Maitén & NM & 706548 & 5596095 & 1,30 \\
\hline “ & Cun-Cun Oeste & $\mathrm{CO}$ & 703088 & 5597452 & 1,40 \\
\hline “ & Anita María & $\mathrm{AM}$ & 697222 & 5595025 & 1,40 \\
\hline “ & Chacaipulli & $\mathrm{CP}$ & 695988 & 5593261 & 1,90 \\
\hline$\omega$ & Balsa Norte & $\mathrm{BN}$ & 695694 & 5592383 & 0,85 \\
\hline 3 & Balsa Sur & $\mathrm{BS}$ & 694813 & 5591543 & 0,61 \\
\hline 6 & El Mosqueto & EM & 694546 & 5590769 & 0,63 \\
\hline 6 & Cuyincahuin & $\mathrm{CU}$ & 693429 & 5586939 & 0,50 \\
\hline 66 & Camping & $\mathrm{CA}$ & 692278 & 5586120 & 0,18 \\
\hline 6 & San Pedro & SP & 691787 & 5586085 & 0,20 \\
\hline 66 & Quinchilca & QI & 692029 & 5586006 & 0,20 \\
\hline
\end{tabular}

\section{RESULTADOS}

El muestreo mensual de las tres macrozonas del río San Pedro, permitió el reconocimiento de 13 especies de peces nativos. Todas las especies presentan marcados patrones temporales de abundancia a lo largo del ciclo anual. Sin embargo, hay especies que presentan sus períodos de máxima abundancia en la estación invernal, coincidentemente con las menores longitudes estándar medias ( $L e)$, debido a la presencia de juveniles del año. Esta situación se constató en las especies: Aplochiton taeniatus Jenyns 1842, Basilichthys australis
Eigenmann 1927, Galaxias maculatus (Jenyns 1842), Galaxias platei Steindachner 1898, Odontesthes mauleanum (Steindachner 1896), Percichthys trucha (Valenciennes 1833) y Percilia gillissi Girard 1854 (Tabla II, Fig. 2). En contraste, hay otras especies que presentan sus máximas abundancias y juveniles del año, en el período de primavera y verano, tales como: Aplochiton zebra Jenyns 1842, Brachygalaxias bullocki (Regan 1908), Cheirodon australe Eigenmann 1927, Diplomystes camposensis Arratia 1987, Geotria australis Gray 1851 y Trichomycterus aerolatus (Valenciennes 1846) (Tabla II, Fig. 3). 
TABLA II. Resumen de los resultados obtenidos en el presente estudio. Las 13 especies nativas presentes en el área de estudio han sido ordenadas según si son más abundantes en el período de invierno o de primavera/verano. Para cada especie se indica el número total de ejemplares estudiados, los períodos en que la especie presentó su mayor abundancia (CPUE) y la mínima longitud estándar (Le) media $(\mathrm{mm})$. Además se indica en la macrozona en que la especie presentó sus mayores abundancias (CPUE) a lo largo del estudio.

TABLE II. Summary of results obtained in this study. The 13 native species present in the study area have been ordered as they are more abundant in the winter period, or spring / summer. For each species, indicating the total number of specimens studied, the periods presented as the most abundant species (CPUE) and the minimum standard length (Le) mean (mm). Also shown in that kind macrozone presented its highest abundances (CPUE) throughout the study.

\begin{tabular}{|c|c|c|c|c|}
\hline Especie & $\begin{array}{l}\text { Número de } \\
\text { ejemplares } \\
\text { (N) }\end{array}$ & $\begin{array}{l}\text { Período } \\
\text { de mayor } \\
\text { abundancia } \\
\text { (CPUE) }\end{array}$ & $\begin{array}{c}\text { Período con } \\
\text { mínima longitud } \\
\text { estándar }(\mathrm{mm})\end{array}$ & $\begin{array}{l}\text { Macrozonas } \\
\text { de mayor } \\
\text { abundancia } \\
\text { (CPUE) }\end{array}$ \\
\hline \multicolumn{5}{|l|}{ a) Más abundantes en invierno } \\
\hline Percichthys trucha Valenciennes 1833 & 662 & Junio-julio & Abril (45) & 1 y 3 \\
\hline Galaxias maculatus (Jenyns 1842) & 4893 & Julio & Diciembre (4) & 1 y 3 \\
\hline Basilichthys australis Eigenmann 1927 & 2589 & Julio & Marzo (48) & 1 y 3 \\
\hline Aplochiton taeniatus Jenyns 1842 & 469 & Julio & Abril (47) & 1 y 2 \\
\hline Odontesthes mauleanum Steindachner 1896 & 12 & Agosto* & Agosto $(45)^{*}$ & 1 y 3 \\
\hline Galaxias platei Steindachner 1898 & 4554 & Agosto & Agosto (46) & 1 \\
\hline Percilia gillissi Girard 1854 & 7984 & Agosto & Julio (35) & 2 \\
\hline \multicolumn{5}{|l|}{ b) Más abundantes en primavera/verano } \\
\hline Brachygalaxias bullocki Regan 1908 & 635 & Octubre & Octubre (23) & 3 \\
\hline Aplochiton zebra Jenyns 1842 & 18 & Octubre* & Diciembre $(60)^{*}$ & 1 \\
\hline Cheirodon australe Eigenmann 1927 & 1798 & Diciembre & Enero (45) & 1 y 3 \\
\hline Trichomycterus aerolatus Valenciennes 1846 & 4489 & Enero-feb. & Enero (35) & 3 \\
\hline Geotria australis Gray 1851 & 1024 & Febrero & Junio (70) & 2 \\
\hline Diplomystes camposensis Arratia 1987 & 1285 & Febrero & Enero (37) & 2 y 3 \\
\hline
\end{tabular}

*Estos datos deben ser considerados con precaución debido al bajo número de ejemplares estudiados.

En cuanto a la abundancia relativa de las 13 especies nativas estudiadas, de los 30.413 ejemplares obtenidos a lo largo de este estudio, las especies más frecuentes fueron P. gillissi (26,3\%), seguidas por G. maculatus (16,1\%), G. platei $(15,0 \%)$ y $T$. areolatus $(14,8 \%)$. Las especies menos frecuentes, fueron $A$. zebra y $O$. mauleanum que estuvieron representadas por escasos individuos a pesar del elevado esfuerzo de muestreo (18 y 12 ejemplares, respectivamente) (Tabla II).

Desde un punto de vista espacial, se registraron claros patrones en la distribución de especies, en las tres macrozonas distribuidas a lo largo de los 40 km del río (M1-M3; Tabla I, Fig. 1). Sólo dos especies presentaron sus mayores abundancias en M1, correspondientes a G. platei y A. zebra (Tabla II, Fig. 4). Estas dos especies también se reportaron en M2 y M3 pero en muy bajas abundancias. M1 se localiza al inicio del área de estudio y es de escasa pendiente y velocidad de la corriente, al igual que M3 localizada aguas abajo (ver pendiente en Tabla I). Sin embargo, ambas se diferencian por el hecho que M1 presenta una conectividad directa con los ensambles de peces del Lago Riñihue, y debiera ser considerada como una zona ecotonal entre comunidades de sistemas lénticos y lóticos. En contraste, M2 corresponde a un sector encajonado del río caracterizado por una mayor pendiente y velocidad media de la corriente $\left(0,42 \pm 0,02 \mathrm{~m} \mathrm{~s}^{-1}\right)$, y por la presencia de una clara secuencia de rápidos/remansos, la cual no existe en M1 y M2. Ninguna especie presentó sus mayores abundancias en este tramo del río (M2) (Tabla II; Fig. 4). Aguas abajo de esta zona del río correspondiente a $\mathrm{M} 3$, sólo $B$. australis destaca por su abundancia, aunque esta especie también está presente en las restantes estaciones (Tabla II, Fig. 4). 

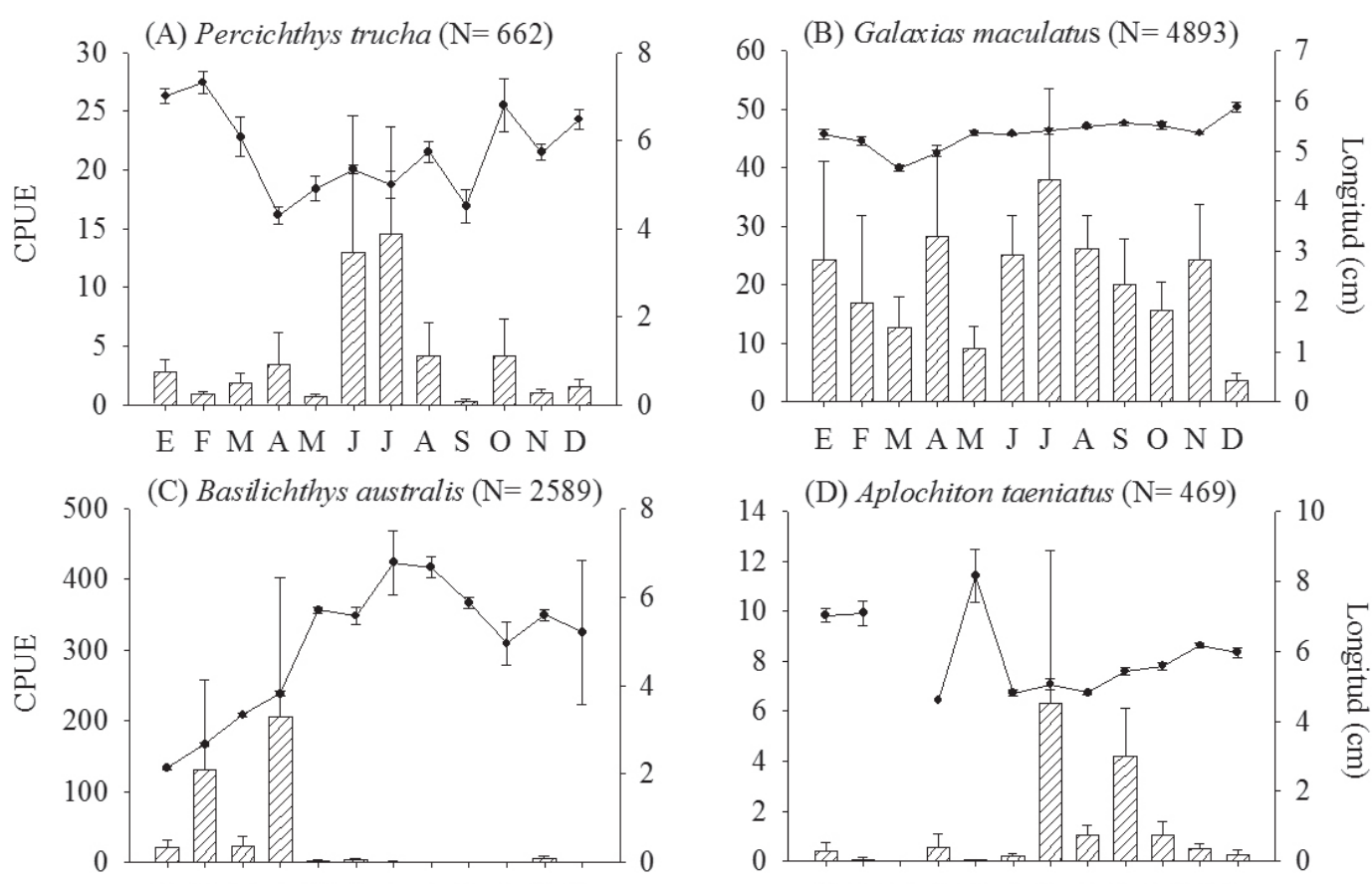

(D) Aplochiton taeniatus $(\mathrm{N}=469)$
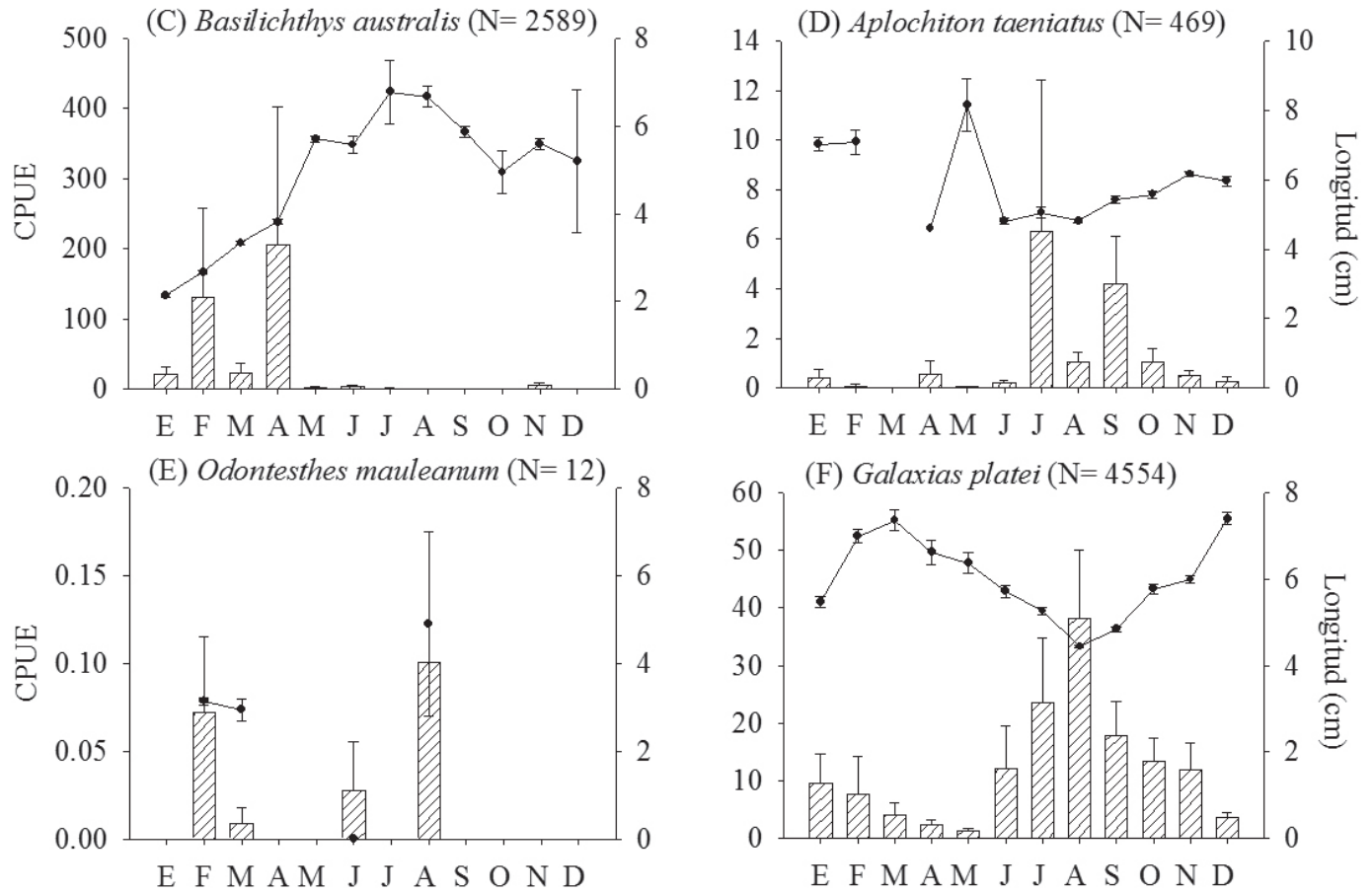

E F M A M J A S O N D
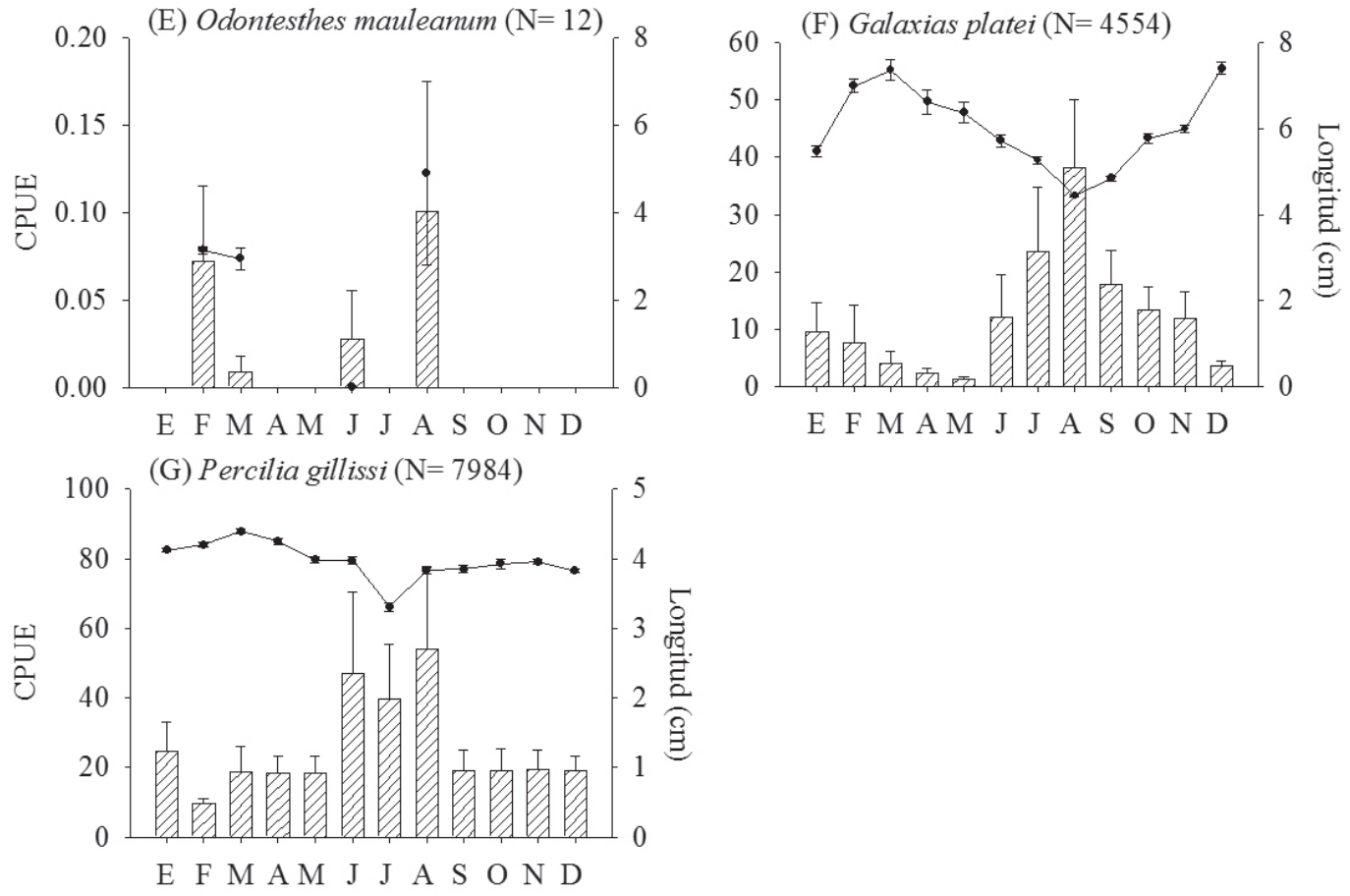

FIgURA 2. Variabilidad temporal de las abundancias de las diferentes especies expresadas como capturas por unidad de esfuerzo (CPUE)

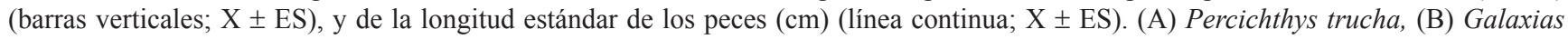
maculatus, (C) Basilichthys australis, (D) Aplochiton taeniatus, (E) Odontesthes mauleanum, (F) Galaxias platei, (G) Percilia gillissi.

FIGURE 2. Temporal variability of the abundances of different species expressed as capture per unit effort (CPUE) (vertical bars; $\mathrm{X} \pm \mathrm{SE}$ ) and standard length of fish (cm) (solid line, $\mathrm{X} \pm \mathrm{SE}$ ). (A) Percichthys trucha, (B) Galaxias maculatus, (C) Basilichthys australis, (D) Aplochiton taeniatus, (E) Odontesthes mauleanum, (F) Galaxias platei, (G) Percilia gillissi. 


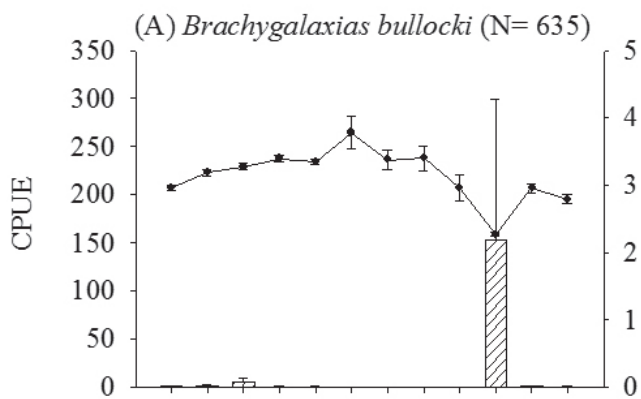

E F M A M J J A S O N D

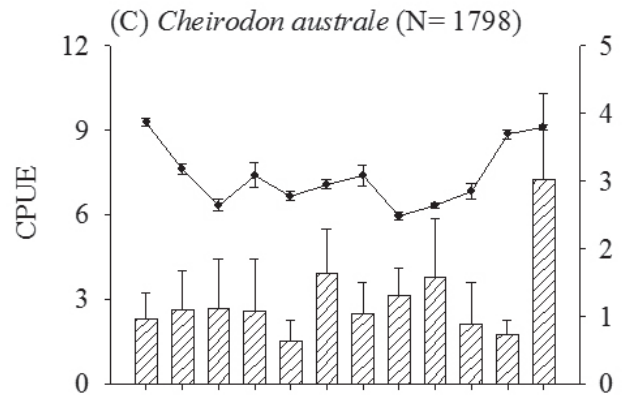

E F M A M J J A S O N D

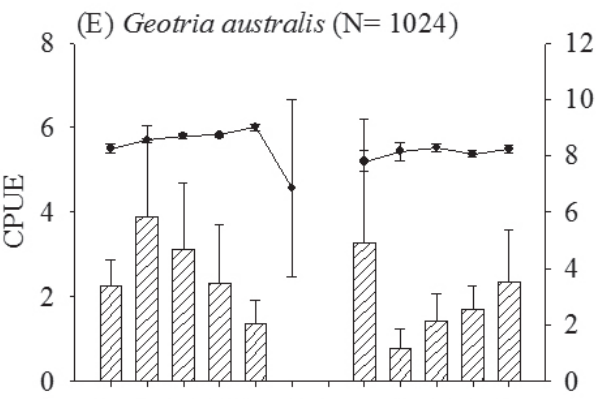

E F M A M J A S O N D
(B) Galaxias maculatus $(\mathrm{N}=4893)$

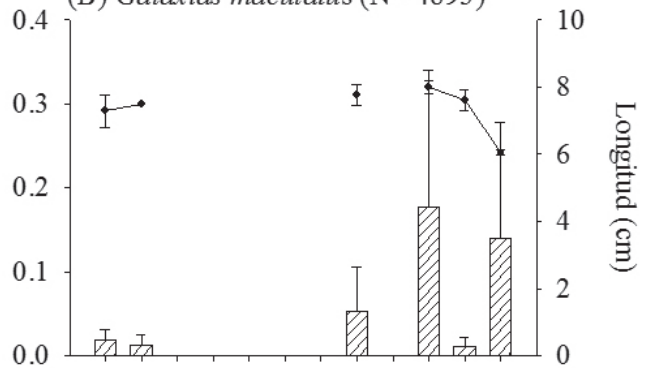

E F M AM J J A S O N D

(D) Trichomycterus areolatus $(\mathrm{N}=4489){ }_{6}$

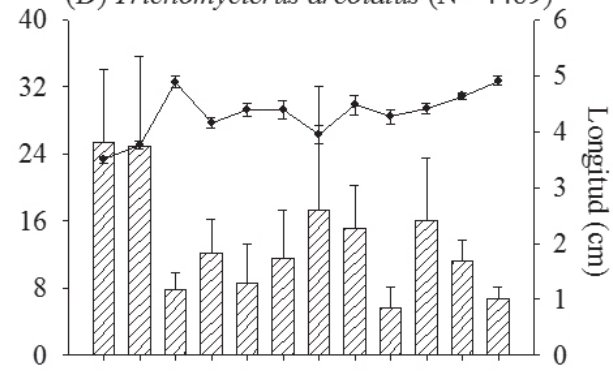

E F M A M J J A S O N D

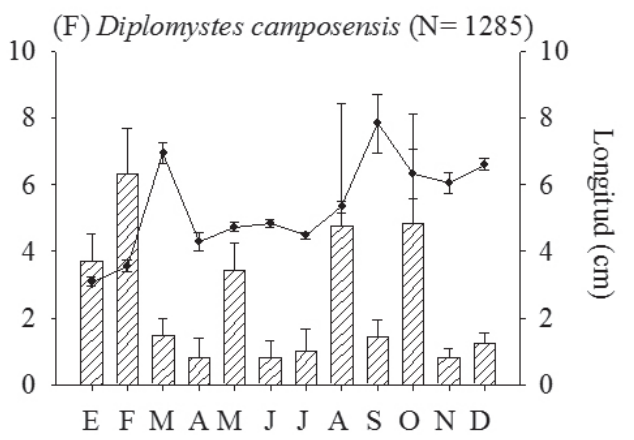

FIgURA 3. Variabilidad temporal de las abundancias de las diferentes especies expresadas como capturas por unidad de esfuerzo (CPUE) (barras verticales; X \pm ES), y de la longitud estándar de los peces (cm) (línea continua; X \pm ES). (A) Blachygalaxias bullocki, (B) Aplochiton zebra, (C) Cheirodon australe, (D) Trichomycterus areolatus, (E) Geotria australis, (F) Diplomystes camposensis.

FIGURE 3. Temporal variability of the abundances of different species expressed as capture per unit effort (CPUE) (vertical bars; $\mathrm{X} \pm \mathrm{SE}$ ) and standard length of fish (cm) (solid line, $\mathrm{X} \pm \mathrm{SE}$ ). (A) Blachygalaxias bullocki, (B) Aplochiton zebra, (C) Cheirodon australe, (D) Trichomycterus areolatus, (E) Geotria australis, (F) Diplomystes camposensis 

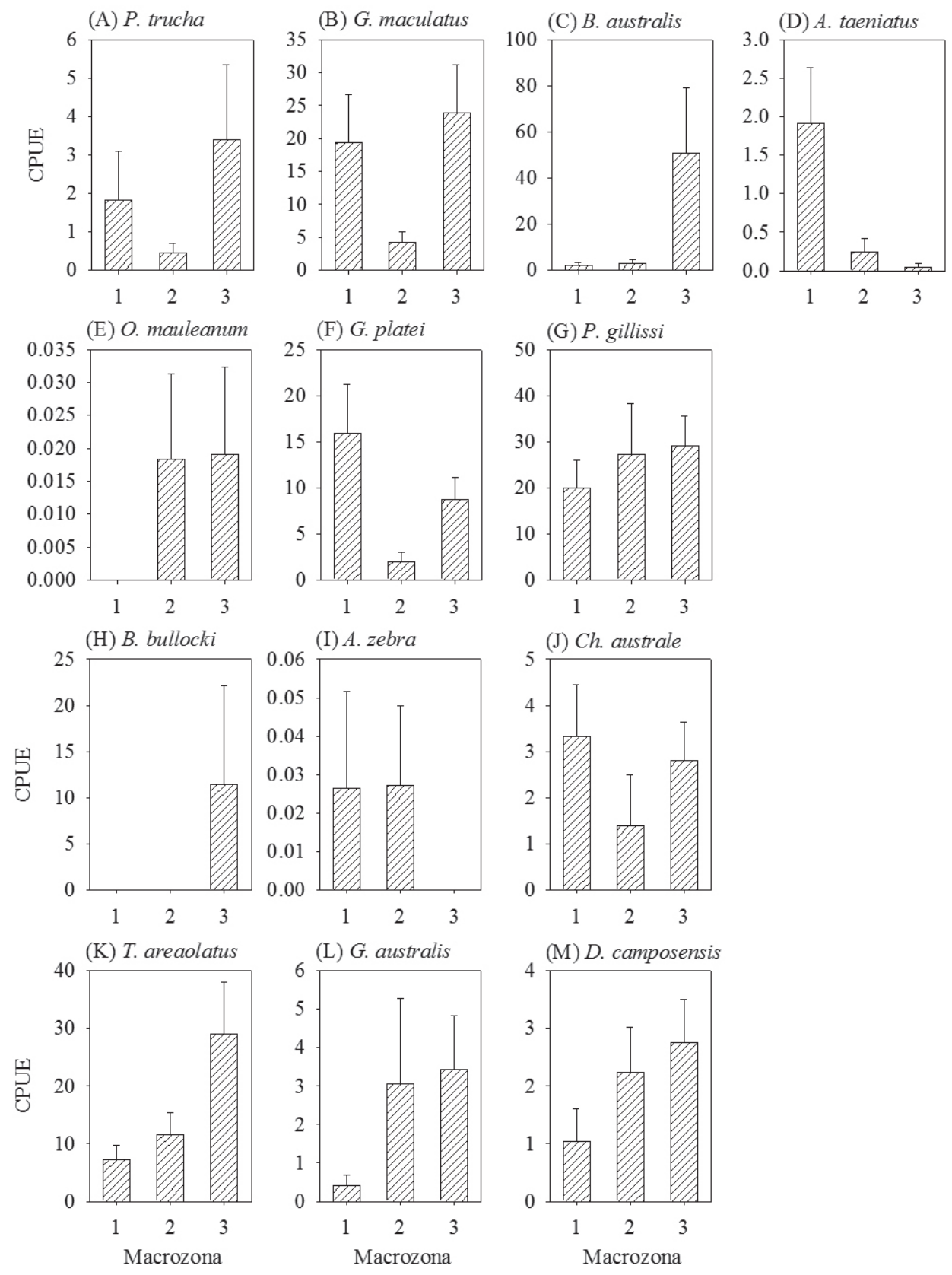

Figura 4. Variabilidad espacial de las abundancias expresadas como captura por unidad de esfuerzo (CPUE), en las tres macro zonas estudiadas en el río san Pedro, Chile (X \pm ES). (A) Percichthys trucha, (B) Galaxias maculatus, (C) Basilichthys australis, (D) Aplochiton taeniatus, (E) Odontesthes mauleanum, (F) Galaxias platei, (G) Percilia gillissi, (H) Blachygalaxias bullocki, (I) Aplochiton zebra, (J) Cheirodon australe, (K) Trichomycterus areolatus, (L) Geotria australis, (M) Diplomystes camposensis.

FIGURE 4. Spatial variability of abundance expressed as capture per unit effort (CPUE) in the three macro areas studied in the San Pedro

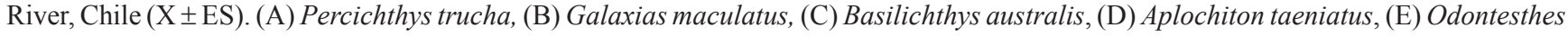
mauleanum, (F) Galaxias platei, (G) Percilia gillissi, (H) Blachygalaxias bullocki, (I) Aplochiton zebra, (J) Cheirodon australe, (K) Trichomycterus areolatus, (L) Geotria australis, (M) Diplomystes camposensis. 
Al observar el patrón longitudinal de las abundancias de todas las especies, se aprecia que cuatro de ellas (P. trucha, $G$. maculatus, $B$. australis y $C h$. australe) son más abundantes en los tramos de baja pendiente y velocidad de la corriente (M1 y M3). Estas especies, aunque también están presentes en M2, siempre muestran abundancias muy bajas en ella, dada su preferencia por aguas más calmas (Tabla II, Fig. 4). De las restantes especies, todas menos $A$. taeniatus y $A$. zebra, mostraron una tendencia a aumentar sus abundancias en el sentido de la corriente, es decir desde M1 a M3. Por el contrario, las dos especies de Aplochiton disminuyeron su abundancia en el mismo sentido longitudinal, presentando mayores abundancias en la zona M1 próxima al Lago Riñihue (Tabla II, Fig. 4).

\section{DISCUSION}

Los resultados obtenidos en el presente estudio, son similares a los obtenidos por Colin et al. (2012) y sugieren que la zona ecotonal lago-río presenta una dinámica muy particular, distinta a la que existe dentro de los lagos o en el curso medio de los ríos. Como se observa en la Tabla II y se discute en el texto, hay especies dentro de las zonas ecotonales de M1 que mantienen una estrecha relación con las poblaciones lacustres (e.g. G. platei), mientras que para otras típicamente fluviales, esta zona representa un límite en su distribución y abundancia río arriba (e.g. D. camposensis).

DiNÁMICA ESPACIO-TEMPORAL EN EL ECOTONO LACUSTREFLUVIAL

Los resultados de este estudio muestran que a pesar de estudiarse un reducido tramo de río (ca. $40 \mathrm{~km})$, es posible observar importantes cambios espaciales en la estructura comunitaria, los cuales estarían asociados fundamentalmente a las condiciones hidromorfológicas de las tres zonas estudiadas y a la influencia del Lago Riñihue. Por otra parte, desde un punto de vista temporal, es posible reconocer claras diferencias en las abundancias y tallas de las especies presentes, las cuales estarían en estrecha relación con la historia de vida de las diferentes especies.

De las cinco especies de Osmeriformes Galaxiidae presentes en el área, i.e. G. maculatus, G. platei, B. bullocki, A. taeniatus y A. zebra, las dos especies del género Aplochiton fueron las que estuvieron menos representadas en el área de estudio. Tanto A. taeniatus como A. zebra, estuvieron presentes preferentemente en las cercanías del Lago Riñihue (M1), lo cual es consistente con los resultados obtenidos por Colin et al. (2012), que señalan mayores abundancias de estas especies aguas arriba del área de estudio. Todos los ejemplares capturados en este estudio fueron juveniles, por lo que es difícil inferir sobre sus épocas reproductivas a partir de las tallas registradas, las que han sido escasamente estudiadas (con la excepción de Campos 1969 y Lattuca et al. 2008). Las otras tres especies estuvieron preferentemente asociadas a aguas calmas, por lo cual estando ausentes o con muy bajas abundancias en M2, caracterizada por aguas con mayor velocidad de la corriente que M1 y M3. Todos los ejemplares de B. bullocki fueron registrados en M3 localizada en la zona baja del área de estudio, en los hábitats característicos descritos para esta especie (Cussac et al. 2004; Habit et al. 2010). La alta abundancia de B. bullocki en el mes de octubre, coincidentemente con una reducción de su talla corporal, sugieren un periodo de reclutamiento poblacional en esa época, lo cual es consistente con lo indicado por McDowall (1971). Por el contrario, en el caso de $G$. platei, a pesar de haber sido registrada en las tres zonas, presentó sus máximas abundancias en M1, lo que sugiere una estrecha relación de estos organismos con los que habitan en el Lago Riñihue. De hecho, G. platei es una especie descrita típicamente como lacustrina (Cussac et al. 2004). En contraste, Galaxias maculatus se mostró más generalista, siendo muy abundante tanto en M1 como en M3, coexistiendo con las demás especies de galáxidos. A pesar que la distribución espacial de G. maculatus se superpone con $G$. platei y $B$. bullocki, sus máximas abundancias en el río tienden a segregarse temporalmente. Los datos de abundancia y de longitud estándar sugieren que a pesar que tanto $G$. platei como $G$. maculatus reclutan en invierno, esta última tendería adelantarse a la segunda en aproximadamente un mes. Otra diferencia aparente es que el período de reclutamiento de G. maculatus es más extenso que el de G. platei. Para ambas especies de Galaxias las mayores abundancias y menores tamaños corporales se obtuvieron en las planicies de inundación que se forman en el período invernal, lo que confirma que éste es un ambiente de crianza de juveniles (ver Montoya et al. 2012). Si bien G. maculatus no muestra diferencias considerables en la frecuencia de tallas durante el año, G. platei registra una caída importante de la talla en agosto. Estos antecedentes sugieren la existencia de dos etapas de reclutamiento para G. maculatus, lo cual concuerda con los dos máximos reportados en el factor de condición, descritos por Cifuentes et al. (2012), en el mismo río y en otros sistemas por Cussac et al. (2004).

Las dos especies de Atheriniformes presentes en el área, i.e. $B$. australis y $O$. mauleanum, presentan marcadas diferencias en sus abundancias. Mientras la primera es abundante, la segunda está prácticamente ausente en el área. En el hecho, solo se capturaron 12 ejemplares de esta última especie durante este estudio. Las dos especies se registraron en las aguas más calmas, tendiendo a aumentar su abundancia en el sentido de la corriente. Para O. mauleanum se ha descrito un lento crecimiento de juveniles en verano en la cuenca del río 
Valdivia, lo que se traduce en que los ejemplares alcancen los $25 \mathrm{~cm}$ de tamaño a los cuatro años de edad (Klink \& Eckman 1985). En relación a B. australis, la mayoría de los ejemplares capturados correspondieron a juveniles (ver Cifuentes et al. 2012). Tomando en consideración la información sobre los huevos de esta especie descritos por Montoya et al. (2012), se sugiere que esta especie utiliza ampliamente las planicies de inundación generadas por crecidas invernales con fines reproductivos, aunque comparativamente en menor grado que G. maculatus. Las dos especies de Siluriformes estudiadas en el área, i.e. $D$. camposensis y $T$. areolatus, estuvieron escasamente representadas en las proximidades del Lago Riñihue (M1), mostrando un claro gradiente positivo de abundancia río abajo (hacia M3). De sus patrones espaciales de abundancia a lo largo del río, estas dos especies se asemejan bastante en los patrones temporales de abundancia, con mayores valores en el período de verano, especialmente en el mes de febrero. La mayor abundancia de reclutas de D. camposensis en M3 durante el mes de enero, sugiere que los hábitats presentes en esta zona son importantes para la reproducción de esta especie, ya que está ausente en los tributarios del río (Habit et al. 2009).

Por otro lado, la especie Ch. australe mostró importantes cambios espaciales a lo largo del río, siendo más abundante en aguas calmas de M1 y M3 que en M2. Esta especie presentó una alta constancia en sus abundancias y tamaños a lo largo del tiempo, con la excepción de diciembre cuando fue mayor. Las mayores abundancias de esta especie se presentaron en sitios de pozas naturales o artificiales (pequeños remansos creados por lugareños para realizar actividades recreativas), como ha sido descrito por Campos (1970).

Con respecto a los Perciformes, la especie $P$. gillissi fue la más abundante de todas las presentes en el tramo de río estudiado, y se distribuyó ampliamente en el área, lo cual es consistente con los resultados Colin et al. (2012). Respecto de los hábitats que $P$. gillissi utiliza, ella aparece más abundantemente en rápidos someros, igualmente está presente en ambientes de pozones y rápidos profundos. Las dos especies de Perciformes, P. gillissi y P. trucha presentaron un claro patrón temporal de abundancias, con un máximo evidente en los meses de invierno (junio a agosto para $P$. gillissi y junio y julio para $P$. trucha), coincidentemente con pequeños tamaños corporales de los organismos. En ambos casos, esto parece estar más asociado a la dificultad de capturar ejemplares de mayor talla en época de alto caudal, que a épocas reproductivas. La época de reproducción de $P$. gillissi ha sido descrita para primavera en el lago Panguipulli (Kilian \& Campos 1969) y a la segunda mitad de la primavera para el caso de $P$. trucha (López-Cazorla \& Sidorkewicj 2011). Desde un punto de vista espacial, $P$. trucha estuvo escasamente representada en la zona de mayor pendiente y velocidad de la corriente (M2). Las mayores abundancias por hábitat se registraron en rápidos someros (García et al 2012). Por otro lado, se constató que $P$. trucha ingresa a las planicies de inundación que se forman en invierno, pero no es un habitante frecuente y abundante de ellas. Lo anterior, sugiere que los principales desplazamientos de esta especie son de tipo laterales entre ambientes profundos y someros.

El ciclóstomo $G$. australis no mostró diferencias estacionales significativas que reflejen una importante dinámica temporal a lo largo del año, ya que sólo se observó una menor abundancia en época de alto caudal. Esta especie sólo fue registrada en estado larvario (fases ammocetes y macroftalmia; ver Neira 1984), con una población que no evidencia una clara estructura de tallas. A lo largo del río registró su mayor abundancia en los sitios en M3, aún cuando también está presente en M1 y M2, pero con una abundancia menor. Esta especie presentó mayores abundancias en ambientes de baja velocidad de corriente y alta depositación de sedimentos, como pozas, siempre asociada a parches fangosos (ver García et al. 2012).

\section{El ecotono Lago Riñinue - Río San Pedro}

Este estudio representa la primera aproximación en Chile al estudio de las zonas ecotonales de sistemas lacustres y fluviales. Los resultados muestran que la estrecha relación del río San Pedro con el Lago Riñihue, hacen que esta zona transicional de la parte alta del río San Pedro, presente características que se diferencian claramente de las existentes río abajo (M2 y M3; Tabla II). Los ecosistemas fluviales como el río San Pedro, se caracterizan por ser heterogéneos y complejos. Estos atributos dependen de fenómenos hidrológicos, y procesos geomorfológicos y ecológicos que delimitan parches de hábitat a través de múltiples escalas (Townsend 1989; Poff et al. 1997; Winemiller et al. 2010; Colin et al. 2012; García et al. 2012). A lo largo de los ríos existen parches con propiedades hidrogeomorfológicas particulares, que se alternan unos con otros determinando una compleja configuración espacial (Naiman et al. 1988; Wu \& Loucks 1995; Poole 2002; Stanford et al. 2005), El concepto de parche, también puede extenderse a la biota, ya que las comunidades pueden ser consideradas como un parche o ensamble, inserto en una matriz con estructura o composición diferente (Winemiller et al. 2010; García et al. 2012). Su tamaño es escala e individuo dependiente y están delimitados por discontinuidades físicas y ecológicas naturales o inducidas por el hombre (Naiman et al. 1988). En los límites de cada uno, existen cambios repentinos que dan lugar a ecotonos dentro del ecosistema fluvial (Naiman et al. 1988). Sin embargo, también surgen ecotonos en la interfase con otros ecosistemas gracias a la multidimensionalidad de 
los ríos (Naiman et al. 1988; Naiman \& Décamps 1997; Winemiller et al. 2010). Los ríos interactúan en cuatro dimensiones con otros componentes del paisaje fluvial. La dimensión longitudinal, dada por las interacciones entre segmentos del cauce principal, la dimensión lateral dada por los flujos entre el canal principal y las planicies de inundación, la dimensión vertical dada por los flujos con el hiporreo y la dimensión temporal (Ward 1989). La transición entre el río y las distintas dimensiones, dan origen a ecotonos entre ecosistemas contrastantes, que pueden ser detectados fácilmente a escalas intermedias. Por ejemplo la dimensión lateral de lagos y ríos, da a lugar un ecotono entre el ambiente acuático y el ambiente terrestre llamado zona ribereña (Naiman \& Décamps 1997; Colin et al. 2011). En esta confluyen elementos de ambos ecosistemas y surgen condiciones ambientales particulares que sustentan procesos y especies únicas (Naiman \& Décamps 1997; Tockner et al. 2010). Lo mismo ocurriría en la dimensión vertical, temporal y longitudinal (Ward et al. 1998). En la dimensión longitudinal de los ríos con regulación lacustre, se formaría un ecotono entre el sistema lacustre y el sistema fluvial. Este depende de la posición dentro de la cuenca, del tamaño y forma del lago así como del tamaño y forma del río (Jones 2010). Esta es una situación compleja en el caso de la cuenca hidrográfica del río Valdivia, tomando en consideración que en esta cuenca existen ocho lagos, i.e. Lácar, Pirehueico, Neltume, Pellaifa, Calafquén, Pullinque, Panguipulli y Riñihue (ver Campos et al. 1978).

En algunos casos lagos y ríos se encuentran íntimamente conectados y sometidos a una fuerte influencia recíproca. Esta interacción podría perturbar los patrones longitudinales predichos por el concepto de River Continnum (Vannote et al. 1980), ya que por la acción reguladora del lago podría generar efectos similares a los de la Discontinuidad Seriada (Ward \& Stanford 1983). En esta interfase ocurren cambios abruptos en características físicas que afectan la estructura y función de las comunidades acuáticas (Jones 2010). A mediana y pequeña escala, la estructura del hábitat experimenta importantes cambios, dados por variaciones en la hidráulica y el sustrato (ver García et al. 2011). El caudal es la variable maestra de los ecosistemas fluviales, porque controla múltiples procesos que fijan la estructura y función ecológica en múltiples escalas (Poff et al. 1997). Las propiedades hidráulicas del caudal determinan el grado de estrés hídrico al que están sometidos los organismos, que afecta directamente su metabolismo y patrones de desplazamiento (Statzner \& Higler 1986; Brooks et al. 2005; Kokes 2011). El tamaño, forma y textura del sustrato, influyen sobre la disponibilidad de refugios para las especies acuáticas y sobre el área disponible para la producción o retención de materia orgánica. La compactación e intersticios del sustrato, pueden ofrecer protección contra el estrés hídrico, la depredación y ofrecen hábitats para las distintas etapas del ciclo de vida de diversas especies. Finalmente, la interacción entre las propiedades físicas del caudal y la arquitectura del sustrato, generan gradientes de profundidad y velocidad que delimitan diferentes parches de hábitat físico (Maddock 1999). En lagos, el hábitat físico está dominado por sedimentos finos, plantas acuáticas y detritus vegetal, sometidos a bajas velocidades de caudal (Schlinder 2002). A medida que el lago se acerca al desagüe (e.g. M1), el ambiente lacustre es reemplazado por un ambiente lótico, dominado por sedimentos de gran tamaño y sometido a altas velocidades (e.g. M2). Este cambio de ambiente determina, una configuración espacial compleja en la transición lagorío y se ve reflejada en la biota (ver Colin et al. 2012).

Por otra parte, los parámetros fisicoquímicos del agua y la productividad muestran fuertes cambios en el caso de los ecotonos embalse-río. Entre el embalse y el río la temperatura puede alcanzar hasta $5^{\circ} \mathrm{C}$ de diferencia y marcar fuertes cambios ecológicos a través de la zona de transición (Rader et al. 2011). Sin embargo, en el caso de los ecotonos lago-río, los parámetros fisicoquímicos tienden a ser más homogéneos por la influencia del lago. Por ejemplo, en el caso del Lago Riñihue y río San Pedro en (M1), el delta de temperatura es inferior a $0,5^{\circ} \mathrm{C}$ (Valdovinos, datos no publicados). Este efecto disminuye aguas abajo por la influencia de los tributarios y los flujos hídricos verticales. La producción primaria autóctona tiende a reducirse desde el lago al río y por lo tanto las fuentes de materia orgánica cambian a través del ecotono. En el lago y bajo condiciones naturales, la principal fuente de materia orgánica y nutrientes es el fitoplancton. Mientras el ecosistema va adquiriendo características lóticas, la fuentes alóctonas de materia orgánica i.e. detritus vegetal, invertebrados terrestres, son más importantes para la productividad del ecosistema acuático (Valdovinos, datos no publicados). Jones (2010), señala que el lago aporta importantes cantidades de materia orgánica particulada fina, promoviendo la abundancia de especies filtradoras. Estas características, determinan la composición taxonómica y funcional de las comunidades en el ecotono, por que controlan el comportamiento $\mathrm{y}$ la organización trófica funcional de los individuos. Por ejemplo, se ha observado que a medida que el aporte de zooplancton lacustre se reduce, la deriva de insectos acuáticos se incrementa, mientras que la abundancia de peces zooplanctívoros se reduce (Willis \& Magnuson 2000). En el caso del sistema estudiado, los aportes de fitoplancton procedente del lago Riñihue explicarían a presencia de importantes poblaciones del bivalvo filtrador Diplodon chilensis (Gray 1828), en el lecho del río de M1 (Valdovinos, datos no publicados).

El ecotono entre lagos y ríos no se restringe solo al ambiente acuático, sino que también puede extenderse al ambiente ribereño, dada la fuerte interacción que existe entre los 
cuerpos de agua dulce y su planicie de inundación. Desde el punto de vista ecológico las planicies son ecotonos o Zonas de Transición Acuática-Terrestre (ATTZ), que albergan múltiples procesos ecológicos. Estos involucran elementos de ambos ambientes y su grado de conectividad depende de los "pulsos de inundación" y "pulsos de caudal" (Junk et al. 1989). Los pulsos de inundación dependen de factores climáticos y son desbordamientos interanuales que inundan completa o parcialmente la planicie de inundación de ríos y lagos (Poff et al. 1997; Dettinger \& Diaz 2000). Mientras que los pulsos de caudal son variaciones intranuales que generan aumentos y descensos de nivel sin abandono del canal principal (Tockner \& Stanford 2002). Esta dinámica hidrológicagenera parches dehábitatribereños caracterizados por bajos niveles de estrés hídrico, altas temperaturas y gran periodo de residencia hídrica (Smock et al. 1992; Tockner et al. 1999; Thorp \& Casper 2003; Hein et al. 2005), que promueven el procesamiento biogeoquímico y recambio de especies (Barth \& Veizer 1999). La estabilidad de los parches ribereños en los ecotonos lago-río, permitiría obtener una mejor aproximación de las interacciones biológicas más relevantes en esta transición (Thorp \& Casper 2002, 2003; Ning et al. 2010a; Ning et al. 2010b; Ning \& Nielsen 2011). Por otra parte, dado que el ambiente ribereño y el río actúan como corredores biológicos, las interacciones y procesos biológicos que ocurran en el ecotono lago-río pueden ser transferidos en todas la dimensiones del sistema fluvial (Vannote et al. 1980).

Finalmente, los resultados de este estudio muestran que el ecotono Lago Riñihue - río San Pedro, corresponde a una de las zonas más diversas en ictiofauna nativa del país, y dado su escaso grado de intervención humana, la hacen ser un adecuado sitio de estudio de ecotonos lacustres - fluviales, y las variaciones espacio - temporales de la biota acuática. Muchas de las especies presentes en este río poseen complejos patrones poblacionales (Victoriano et al. 2012), incluso dentro de una misma especie (e.g. Galaxias maculatus), constituyendo distintas unidades naturales que se desarrollan diferencialmente entre las diferentes zonas del río (ver García et al. 2012; Colin et al. 2011; Montoya et al. 2012). Es posible observar importantes cambios espaciales en la estructura comunitaria en un reducido tramo de río, los cuales estarían asociados fundamentalmente a las condiciones hidromorfológicas de las tres zonas estudiadas y a la marcada influencia del Lago Riñihue. Por otra parte, desde un punto de vista temporal, es posible reconocer claras diferencias en las abundancias y tallas de las especies presentes, las que estarían en estrecha relación con la historia de vida de las diferentes especies. Los resultados de este estudio permiten visualizar la relevancia de los ecotonos lacustres - fluviales, en un contexto funcional y de conservación de la biodiversidad dulceacuícola.

\section{AGRADECIMIENTOS}

Los autores agradecen a COLBUN S.A. por el financiamiento de este estudio y al proyecto DIUC Semilla Patagonia 210.310.057-1SP por el financiamiento de la publicación. A Catalina Puga del Hotel Riñimapu y propietarios de predios aledaños al río San Pedro, por su importante colaboración en la realización del trabajo de campo a lo largo de todos los años de este estudio. Igualmente se agradece a todas las personas que directa o indirectamente participaron en la elaboración de este estudio. Finalmente se agradece al proyecto Fondecyt 1110441 que permitió la finalización de este manuscrito.

\section{BIBLIOGRAFÍA}

Allan, D. \& M. Castillo. 2007. Stream ecology, structure and function of running waters. 2da Ed. Springer, New York. $436 \mathrm{pp}$.

Arunachalam, M. 2000. Assemblage structure of stream fishes in the Western Ghats (India). Hydrobiologia 430:1-31.

Barth, J. A. C. \& J. Veizer. 1999. Carbon cycle in St. Lawrence aquatic ecosystems at Cornwall (Ontario), Canada: seasonal and spatial variations. Chemical Geology 159:107-128.

Brooks, A., T. Haeusler, I. Reinfelds \& S. Williams. 2005. Hydraulic microhabitats and the distribution of macroinvertebrate assemblages in riffles. Freshwtaer Biology 50:331-344.

Campos H. 1969. Reproducción de Aplochiton taeniatus Jenyns. Boletín del Mueso Nacional de Historia Natural, 29: 207-222.

CAmpos, H. 1970. Introducción de especies exóticas y su relación con los peces de agua dulce de Chile. Not. Mens. Mus. Nac. Hist. Nat, Chile, 14(162): 3-9.

Campos, H., J. Arenas, W. Steffen \& G. Aguero. 1978. Physical and chemical limnology of Lake Rinihue (Valdivia, Chile). Archiv fur Hydrobiologie 84:405-429.

Cifuentes, R., J. González, G. Montoya, A. Jara, N. Ortíz, P. Piedra \& E. Habit. 2012. Relación longitud-peso y factor de condición de los peces nativos del río San Pedro (cuenca del río Valdivia, Chile). Gayana 76 (Número Especial): 101-110.

Colin, N., P. Piedra \& E. Habit. 2012. Variaciones espaciales y temporales de las comunidades ribereñas de peces en un sistema fluvial no intervenido: río San Pedro, cuenca del río Valdivia (Chile). Gayana 76 (Número Especial): 24-35.

Cussac V., S. Ortubay, G. Inglesias, D. Milano, M. Lattuca, J.P. Barriga, M. Battini \& M. Gross. 2004. The distribution of South American galaxiid fishes: the role of biological traits and post-glacial history. Journal of Biogeography 31: 103-121.

Dettinger, M. D. \& H. F. Diaz. 2000. Global characteristics of stream flow seasonality and variability. Journal of Hydrometeorology 1:289-310.

Dudgeon, D., A. H. Arthington, M. O. Gessner, Z.I. Kawabata, D. J. Knowler, Ch. Leveque, R.J. Naiman, A.H. Prieur- 
Richard, D. Soto, M. L. J. Stiassny \& C. A. Sullivan. 2006. Freshwater biodiversity: importance, threats, status and conservation challenges. Biological Reviews 81:163-182.

Eula. 2007. Línea base central hidroeléctrica río San Pedro. Informe técnico elaborado por el Centro de Ciencias Ambientales EULA, de la Universidad de Concepción. $285 \mathrm{pp}$.

García, A., J. GonzÁlez \& E. Habit. 2012. Caracterización del hábitat de peces nativos en el río San Pedro (cuenca del río Valdivia, Chile). Gayana 76 (Número Especial): 3644.

Habit, E., B. Dyer \& I. Vila. 2006a. Estado de conocimiento de los peces dulceacuícolas de Chile. Gayana (Zoología) 70:100-113.

Habit, E., M. Belk, R. Tuckfield \& O. Parra. 2006b. Response of the fish community to human-induced changes in the Biobío River in Chile. Freshwater Biology, 51(1): 1-11.

Habit, E. M. Belk \& O. Parra. 2007. Response of the riverine fish community to the construction and operation of a diversion hydropower plant in central Chile. Aquatic Conservation: Marine and Freshwater Ecosystems. 17:37-49.

Habit, E. A. Jara, N. Colin, A. Oyanedel, P. Victoriano, J. Gonzalez \& K.Solis-Lufí. 2008. Threatened fishes of the world: Diplomystes camposensis Arratia, 1987 (Diplomystidae) Environmental Biology of Fishes. 84:393-394.

Habit, E., Piedra, P., Ruzzante, D., Walde, S., Belk, M., Cussac, V., Gonzalez, J. \& N. Colin. 2010. Changes in the distribution of native fishes in response to introduced species and other anthropogenic effects. Global Ecology \& Biogeography. 19: 697 - 710

Heegaard, E., A. Lotter \& H. Birks. 2006. Aquatic biota and the detection of climate change: Are there consistent aquatic ecotones? Journal of Paleolimnology 35:507-518.

Hein, T., W. Reckendorfer, J. H. Thorp \& F. Schiemer. 2005. The role of slackwater areas for biogeochemical processes in rehabilitated river corridors: examples from the Danube. Archiv für Hydrobiologie Supplementband 155:425442.

JONES, N. E. 2010. Incorporating lakes within the river discontinuum: longitudinal changes in ecological characteristics in streamlake networks. Canadian Journal of Fisheries and Aquatic Sciences 67:1350-1362.

Junk, W., P. Bayley \& R. Sparks. 1989. The flood pulse concept in river floodplain systems. En: Proceedings of the International Large River Symposium. Canadian Special Publication of Fisheries and Aquatic Sciences 106 :110127. Departament of Fisheries and Oceans, Ottawa, Canadá.

Kilian, A. \& H. Campos. 1969. Súbwissenschwämme als Or. Der Brutpflege eines Fisches. Naturwissenschaften 56(69): 333-334.

KLINK, A. \& R. EcKmann. 1985. Age and growth, feeding habits and reproduction of Cauque mauleanum Steindachner 1986 (Pisces: Atherinidae) in southern Chile. Studies on Neotropical Fauna and Enviroment 20: 239-249.

KoKes, J. 2011. River channel habitat diversity (RCHD) and macroinvertebrate community. Biologia 66:328-334.
Lattuca, M., D. Brown, L. Castiñeira, M. Renzi, C. Luizon, J. URBANSKI \& V. CuSSAC. 2008. Reproduction of landlocked Aplochiton zebra Jenyns (Pisces, Galaxiidae). Ecology of Freshwater Fish 17: 394-405.

López-Carzola, A. \& N. Sidorkewicj. 2011. Age, growth and reproduction in creole perch (Percichthys trucha) in the Negro River, Argentinean Patagonia. Journal of Applied Ichthyology. 27: 30-38 pp.

MADDOCK, I. 1999. The importance of physical habitat assessment for evaluating river health. Freshwater Biology 41:373391.

McDowall, R.M. 1971. The galaxiid fishes of South America. Zoological Journal of the Linnean Society. 50: 33-73.

Montoya, G., A. Jara, K. Solis-Lufí, N. Colin \& E. Habit. 2012. Primeros estadíos del ciclo de vida de peces nativos del río San Pedro (cuenca del río Valdivia, Chile). Gayana 76 (Número Especial): 86-100.

Naiman, R. J. \& H. DÉCAMPS. 1997. The ecology of interfaces: riparian zones. Annual Review of Ecology and Systematics 28:621-658.

Naiman, R. J., H. Décamps, J. Pastor \& C. A. Johnston. 1988. The potential importance of boundaries of fluvial ecosystems. Journal of the North American Benthological Society 7:289-306.

NeIRA, F. 1984. Biomorfología de las lampreas parásitas chilenas Geotria australis Gray, 1851 y Mordacia lapicida (Gray, 1851) (Petromyzontiforms). Gayana (Zoología) 48: 340.

Ning, N. \& D. NiELSEN. 2011. Community structure and composition of microfaunal egg bank assemblages in riverine and floodplain sediments. Hydrobiologia 661:211-221.

Ning, N.S.P., D.L. Nielsen, T.J. Hillman \& P.J. Suter. 2010 a. The influence of planktivorous fish on zooplankton communities in riverine slackwaters. Freshwater Biology 55:360-374.

Ning, N.S.P., D.L. Nielsen, W.L. Paul, T.J. Hillman \& P.J. Suter. 2010b. Microinvertebrate dynamics in riverine slackwater and mid-channel habitats in relation to physico-chemical parameters and food availability. River Research and Applications 26:279-296.

Poff, N. L. R., J. D. Allan, M. B. Bain, J. R. Karr, K. L. Prestegaard, B. D. Richter, R. E. Sparks \& J. C. Stromberg. 1997. The natural flow regime. Bioscience 47:769-784

Poole, G.C. 2002. Fluvial landscape ecology: addressing uniqueness within the river discontinuum. Freshwater Biology 47:641-660.

Rader, R.B., M.C. Belk, R. Hotchisiss \& J. Brown. 2011. The stream-lake ecotone: Potential habitat for juvenile endangered June Suckers (Chasmistes liorus). Western North American Naturalist 70:553-561.

Samways, M. J. \& D. A. B. Stewart. 1997. An aquatic ecotone and its significance in conservation. Biodiversity and Conservation 6:1429-1444.

Smock, L., J. Gladden, J. Riekenberg, L. Smith \& C. Black. 1992. Lotic macroinvertebrate production in three dimensions: Channel surface, hyporheic, and floodplain environments. Ecology 73:876-886.

Stanford, J., M. Lorang, F. Hauer \& J. Jones. 2005. The shifting habitat mosaic of river ecosystems. Verhandlungen 
Internationaler Vereinigung für Limnologie 29: 1-14.

Statzner, B. \& B. Higler. 1986. Stream hydraulics as a major determinant of benthic invertebrate zonation patterns. Freshwater Biology 16:127-139.

Thomasson, K. 1963. Araucanian Lakes. Plankton studies in North Patagonia with notes on terrestrial vegetation. Acta Phtytogeographica Suecica 47: 1- 141.

Thorp, J. H. \& A. F. CASPER. 2002. Potential effects on zooplankton from species shifts in planktivorous mussels: a field experiment in the St Lawrence River. Freshwater Biology 47:107-119.

THORP, J. H. \& A. F. CASPER. 2003. Importance of biotic interactions in large rivers: an experiment with planktivorous fish, dreissenid mussels and zooplankton in the St. Lawrence River. River Research and Applications 19:265-279.

TOCKNER, K. \& J. StANFord. 2002. Riverine flood plains: present state and future trends. Enviromental Conservation 29:308-330.

Tockner, K., D. Pennetzdorfer, N. Reiner, F. Schiemer \& J. V. WARD. 1999. Hydrological connectivity, and the exchange of organic matter and nutrients in a dynamic river-floodplain system (Danube, Austria). Freshwater Biology 41:521-535.

Tockner, K., M. S. Lorang \& J. A. Stanford. 2010. River flood plains are model ecosystems to test general hydrogeomorphic and ecological concepts. River Research and Applications 26:76-86.

Townsend, C. R. 1989. The patch dynamics concept of stream community ecology. Journal of the North American Benthological Society 8:36-50.

Vannote, R. L., G. W. Minshall, K. W. Cummins, J. R. Sedell
\& C. E. Cushing. 1980. The river continuum concept. Canadian Journal of Fisheries and Aquatic Sciences 37:130-137.

Victoriano, P., I. Vera, V. Olmos, M. Dib, B. Insunza, C. MuñozRamírez, R. Montoya, A. Jara \& E. Нabit. 2011. Patrones idiosincráticos de diversidad genética de peces nativos del río San Pedro (cuenca del río Valdivia), un sistema de la región glaciada del sur de Chile. Gayana (Zoología) (en prensa).

WARD, J. 1989. The four-dimensional nature of lotic ecosystems. Journal of the North American Benthological Society $8: 2-8$.

Ward, J. V. \& J. A. Stanford. 1983. The serial discontinuity concept of lotic ecosystems, En: The Dynamics of Lotic Ecosystems, T.D. Fontaine \& S.M. Bartell (eds.), pp. 2942.Ann Arbor Science, Ann Arbor.

Ward, J., G. Bretschko, M. Brunke, D. Danielopol, J. Gibert, T. Gonser \& A. HiLdRew. 1998. The boundaries of river systems: the metazoan perspective. Freshwater Biology 40:531-569.

Willis, T. V. \& J. J. Magnuson. 2000. Patterns in fish species composition across the interface between streams and lakes. Canadian Journal of Fisheries and Aquatic Sciences 57:1042-1052.

Winemiller, K.O., A.S. Flecker \& D.J. Hoeinghaus. 2010. Patch dynamics and environmental heterogeneity in lotic ecosystems. Journal of the North American Benthological Society 29:84-99.

Wu, J. \& O. L. LouCKs. 1995. From balance of nature to hierarchical patch dynamics: a paradigm shift in ecology. Quarterly Review of Biology 70(4): 439-466.

Recibido: 02.01.12

Aceptado: 27.02.12 